\title{
Scanning thermal testing of subsurface defects in metal - coating structures
}

\author{
by A. Chulkov*, B. Oswald-Tranta**, C.Tusch"**, V. Vavilov* \\ * National Research Tomsk Polytechnic University, 634050 Tomsk, Lenin Av., 30, chulkovao@tpu.ru \\ ${ }^{* *}$ Chair of Automation, University of Leoben, Peter-Tunnerstr.27, 8700 Leoben, Austria, \\ beate.oswald@unileoben.ac.at
}

\begin{abstract}
In this study, two line-scanning thermal non-destructive testing procedures using optical and inductive heating are compared in the inspection of metal-non-metal structures. It is demonstrated that, in the case of inductive stimulation, onesided test procedure converts into two-sided by heating the metal through non-metal. With such technique, deep interlayer delaminations can be detected more efficiently than by using surface optical heating.
\end{abstract}

\section{Introduction}

Two-layer structures including a metallic base and thermal insulation coating are ubiquitous in the aerospace, petro chemistry and power production. Thickness of both metallic and non-metallic layers can vary from fractions of millimeter to several millimeters depending on structure purpose, and the most typical defects are delaminations between metal and thermal insulation. Besides, in some cases, like trunk pipe lines, there may be additional construction elements, such as piping shrink sleeves, where additional delaminations between different non-metals may appear. Due to high uniform emissivity of thermal insulation, thermal nondestructive testing (TNDT) is a convenient inspection tool for detecting defects of large lateral size if defect depth does not exceed some millimeters, while thick insulation layers can hardly be tested because of low depth of penetration of thermal energy. In this case, a typical heater includes conventional halogen lamps with the power of 1-4 kW. However, according to the authors' experience, by using large linear heaters, it is possible to achieve inspection productivity of up to $25 \mathrm{sq}$. $\mathrm{m}$. (in the inspection of big cylindrical objects). In this study, some peculiarities of using induction heaters instead of optical are analyzed [1]. A principle advantage of the proposed heating method is that, as a matter of fact, a one-sided TNDT procedure is replaced with a two-sided one thus enhancing detectability of deeper defects.

In TNDT, line induction heating was first applied by Green for evaluating the quality of nuclear fuel elements [2]. In the 1980s, line-scanning optical heating was used in the former USSR to check large-size cylindrical shells made of glass fiber reinforced plastic (GFRP) [3]. In the next decades, the line-scanning TNDT method somewhat lost its attractiveness due to the appearance of high-quality economic infrared (IR) imagers and flexible optical heaters allowing the inspection of large-size objects in the area-by-area mode. Moreover, one-sided pulsed TNDT procedures are more appropriate for performing defect characterization than two-sided procedures. This is explained by a strong dependence of surface temperature signals on defect depth in one-sided TNDT. Nevertheless, in the last decade, the interest to linescanning TNDT has been growing along with the appearance of robotized inspection systems and development of defect characterization algorithms. Results of line-scanning TNDT can be presented in two ways: either related to the inspection device or to the test object itself. In the latter case, thermal distributions on the surface of a test sample can be evaluated at various time delays in a single test session by selecting the corresponding lines from subsequent IR thermograms. Synthetic IR images can be properly processed to allow defect characterization; such algorithms are under development [4].

An example of a TNDT robotized system developed at Tomsk Polytechnic University for the inspection of large-size cylindrical objects is shown in figure 1. The system includes two tubular heat sources (electrical resistive heaters), having the length of $1.1 \mathrm{~m}$ and the total power of $2 \mathrm{~kW}$, and an IR module Optris, both mounted on a robotized manipulator. The IR imager monitors a certain area on the surface of the test object with a time delay in regard to the heating period which depends on the scanning speed and material thermal properties, as well an expected defect depths [4]. An object to be tested rotates with a certain speed. After the TNDT device finishes monitoring a circular segment on the object surface, it moves along the object to scan the next segment, etc. The described system allows continuous inspection of cylindrical objects with a unique productivity of up to $25 \mathrm{sq}$. $\mathrm{m}$. A result of inspection is a panoramic evolution of surface temperature distribution, and the total size of stored data may reach $80 \mathrm{~GB}$ thus requiring to apply automated data processing. 


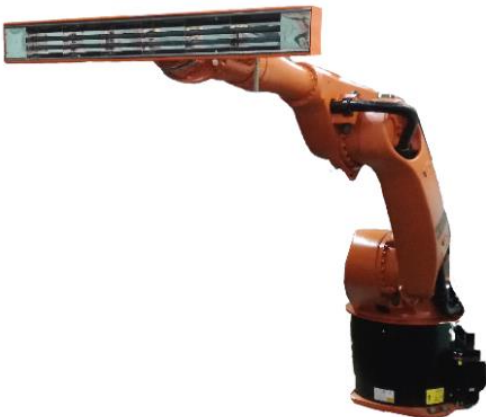

a)

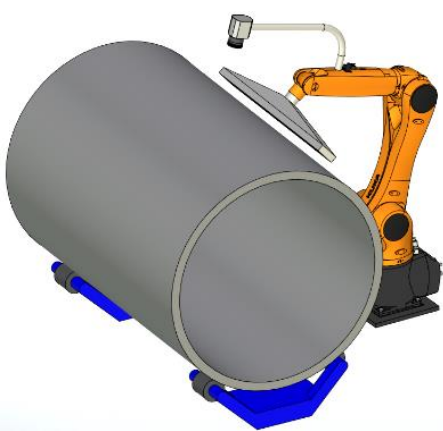

b)

Fig. 1. Robotized thermal NDT system for inspecting large-size cylindrical objects by using line scanning: $a-$ system photo, $b-3 D$ model

The goal of this research was to improve line-scanning TNDT methods for detecting subsurface defects, such as interlayer delaminations which might appear between thermal insulation layers and between the insulation and metallic base. The two different thermal stimulation techniques, namely, optical and inductive, have been quantitatively compared by using the concept of signal-to-noise ratio (SNR). The main advantage of induction heating is that it is applied to the metal through the thermal insulation layer thus converting the one-sided test procedure into two-sided. It is known that, under equal test conditions, a two-sided procedure is typically more efficient than one-sided, especially, if defects can be located through the sample or it is deeply in the thermal insulation counting from the front surface. The disadvantage of such approach is that a two-sided procedure can hardly be used for characterizing defects because varying defect depth weakly influences surface signals.

\section{Test sample}

The following samples with artificial defects were developed. The $3 \mathrm{~mm}$-thick sample base layer was made of Aluminium alloy D16, and two plates of Plexiglas, each with the thickness of $3 \pm 0.3 \mathrm{~mm}$, were used for modelling the thermal insulating coating (note that Plexiglas has similar thermal properties to many types of thermal insulation coatings). In the Plexiglas plates flat bottom holes with the diameter of $10 \mathrm{~mm}$ were drilled in order to simulate subsurface air-filled defects which in real cases occur between the insulation layer and metallic base. The height of bottom holes varied from 0.5 to 1.5 $\mathrm{mm}$, see figure 2. The two Plexiglas plates were glued to each other and to the metallic plate by means of an epoxy adhesive. At the top layer of the sample a group of small drilled holes (green squares in figure 1) simulates the roughness of the surface. Because of the transparency of Plexiglas, the sample was black-painted with an acrylic dye thus having increased its emissivity up to 0.97 .
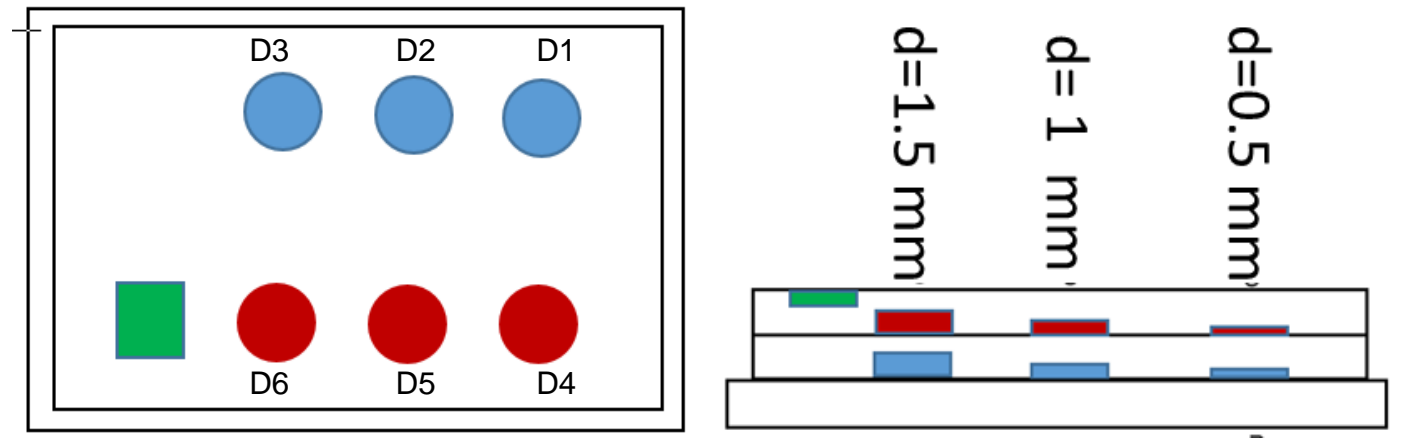

Fig. 2. Scheme of the sample with artificial defects; left: top view, right: side view

The ThermoCalc-3D software from Tomsk Polytechnic University intended for 3D numerical modelling of TNDT problems was used to optimize both heating and observation times. The modelled heating duration was $5 \mathrm{~s}$ with pulse density $2 \mathrm{~kW} / \mathrm{m}^{2}$. Figure 3 shows the calculated results for heating power of $2 \mathrm{~kW} / \mathrm{m}^{2}$ and heat pulse duration of $5 \mathrm{~s}$. An example of the experimental results is illustrated with figure 4 showing the Fourier phasegram in the case of flash heating ( $6 \mathrm{~kJ}, 1 \mathrm{~ms}$ heat pulse, one-sided test procedure). 

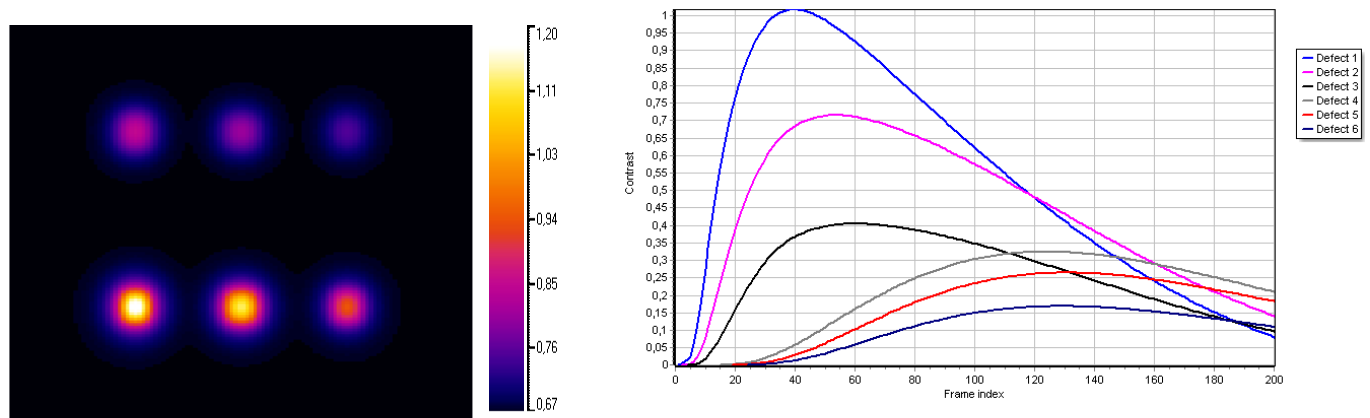

Fig. 3. Numerical simulation results: left - IR thermogram at $80 \mathrm{~s}$, right-evolution of running temperature contrast $\Delta T / T$ for all six defects

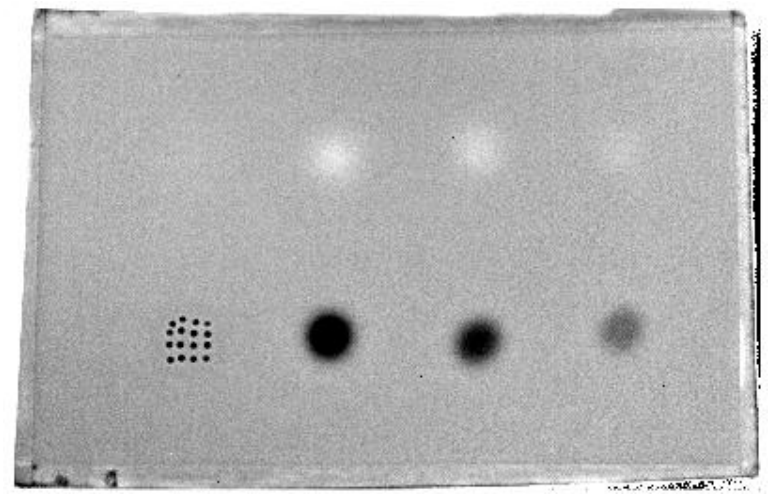

Fig. 4. Experimental results: Fourier phasegram, one-sided flash TNDT

\section{Line-scanning TNDT}

Figure 5 shows two experimental setups used for optical ( $2 \mathrm{~kW}$ halogen lamp) and induction line heating. In all cases, both the IR imager and the heater were placed on the Plexiglas side to implement one-sided line-scanning TNDT. To be more precise, the reflection mode was provided when using the optical heating, while the inductor first generated the Joule heat in the metallic plate which, in its turn, stimulated the thermal insulation, thus realizing the transmission (twosided) procedure. In all cases, the temperature was recorded by means of a FLIR sc7700 IR imager (optical stimulation, 7-13 $\mu \mathrm{m}$ cooled detector) and an IRCAM VELOX 1310k SM IR imager (inductive stimulation, 1.5-5.5 $\mu \mathrm{m}$ cooled detector).

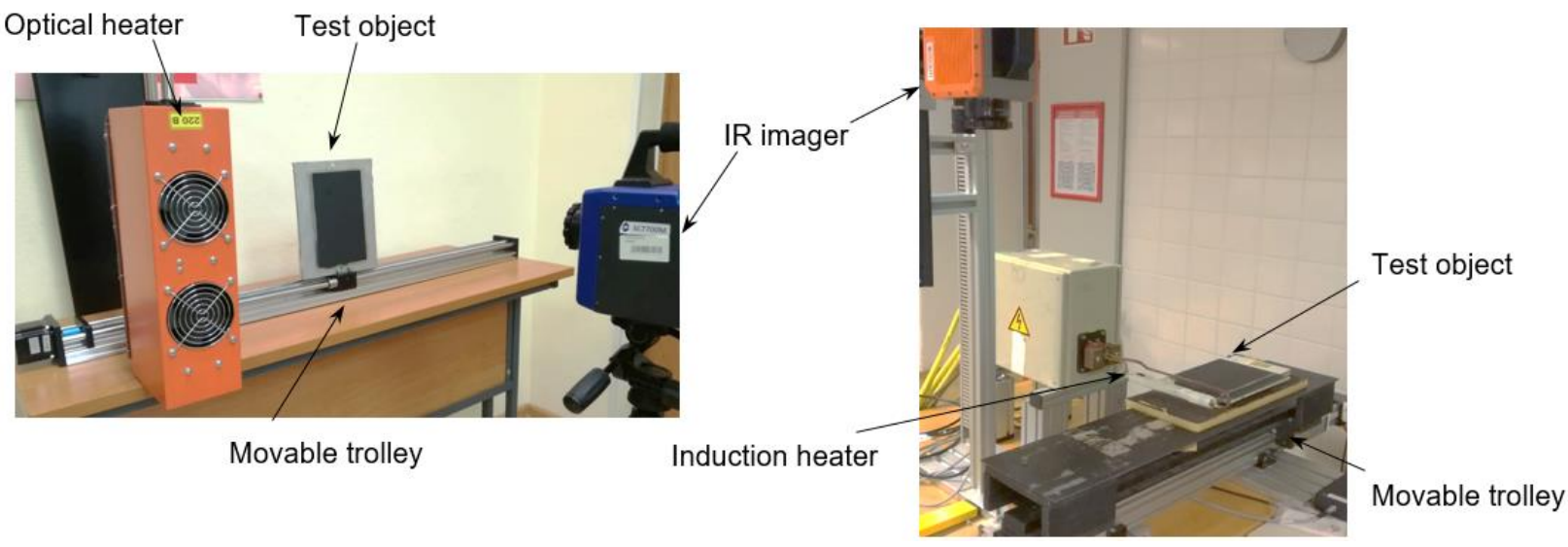

a - setup based on optical heating

$b-$ setup based on inductive heating

Fig. 5. Experimental setups for line-scanning TNDT

Experimental results are presented in figure 6. IR image sequences were obtained by applying line-by-line reconstruction of raw sequences (by keeping the same time delay for each consecutive line). In the case of optical stimulation, the results corresponded to the cooling stage of the thermal process in the time range from 109 to $202 \mathrm{~s}$. The 
sample cooling after inductive heating was analyzed within the $94-160$ s period. The time intervals above were determined experimentally to provide optimum observation times for all defects.

To compare results obtained by using different types of heating, a SNR criterion was applied. The defect and nondefect areas chosen for calculating SNR are shown in figure $6 a, b$ with red and green circles respectively. The evolutions of SNR values are plotted in figure $6 \mathrm{c}, \mathrm{d}$. Before calculating SNR, the IR image sequences were normalized by amplitude with digital levels (DL) being in the range from 0 to 1 . The images were filtered in space and time with median filters.

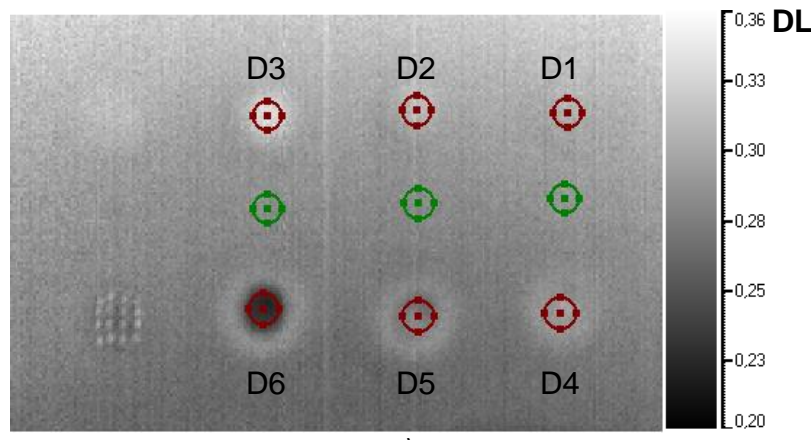

a)

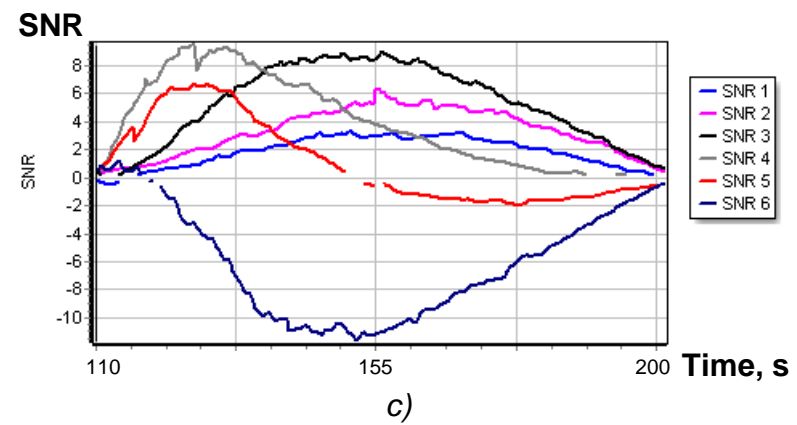

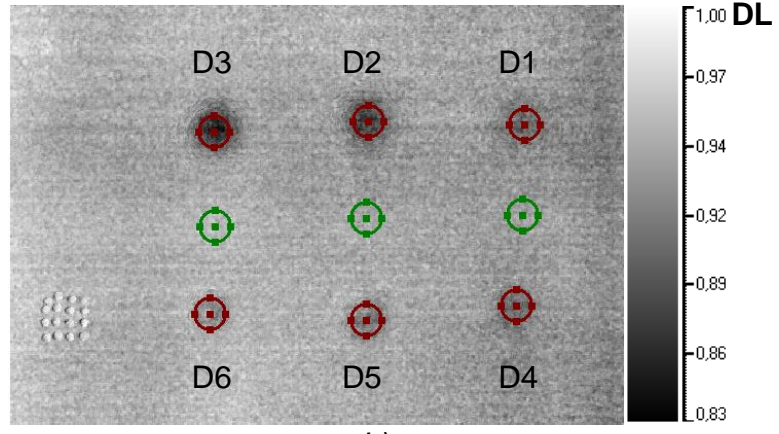

b)

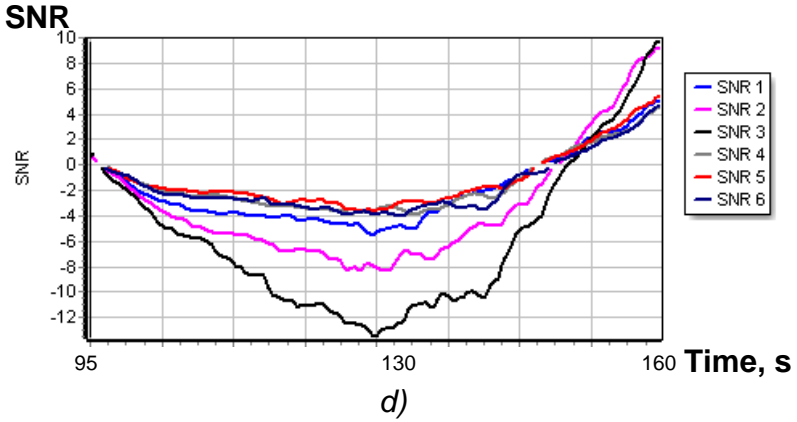

Fig. 6. Experimental results of line-scanning TNDT by applying optical and inductive heating:

a - IR thermogram at $44 \mathrm{~s}$ (optical heating),

$b-I R$ thermogram at $40 \mathrm{~s}$ (inductive heating),

$c-S N R$ evolution in time for D1-D6 defects (optical heating),

$d-S N R$ evolution in time for D1-D6 defects (inductive heating)

The results obtained can be explained in the framework of the classical TNDT theory. One-sided TNDT is very efficient for shallow defects; in this case, defects produce high temperature signals which are weakly subjected to heat diffusion, while temperature "footprints" of deeper defect appear with a longer time delay and "smash" in space. In some cases, shallow defects may reveal a phenomenon of signal inversion where a defect becomes colder than the background because of quick cooling of the above-defect layer. Respectively, deeper defects can be easier detected in a two-sided procedure of which results are weakly dependent on defect location within a test object. For example, under inductive heating, the defects D1-D3 are characterized by the SNR values of 5.8, 8.3 and 13.6, while optical heating provide the values of 3.1, 6.1 and 8.9 respectively. Oppositely, the shallow defects D4-D-6 are characterized by the SNR values from 4 to 6 in the case of inductive heating and from 8 to 12 in the case of optical heating (note that the signal from the defect D6 experiences inversion). When comparing the results obtained with two different types of heating, it is worth remembering that one-sided TNDT procedures are more convenient for performing defect characterization, mainly, due to the fact that surface temperature signals strongly depend on defect depth (figure 6c).

Inductive heating has seemed to be more uniform being independent of thermal insulation optical absorptivity. Surprisingly, the defect "footprints" obtained with inductive stimulation corresponded better to true lateral size of defects. This statement is illustrated with two Fourier phasegrams in figure 7 where defect indications are more consistent with the real defects, at least visually, in the case of inductive heating. This may be explained by stronger heat diffusion around deeper defects in the case of optical heating. 


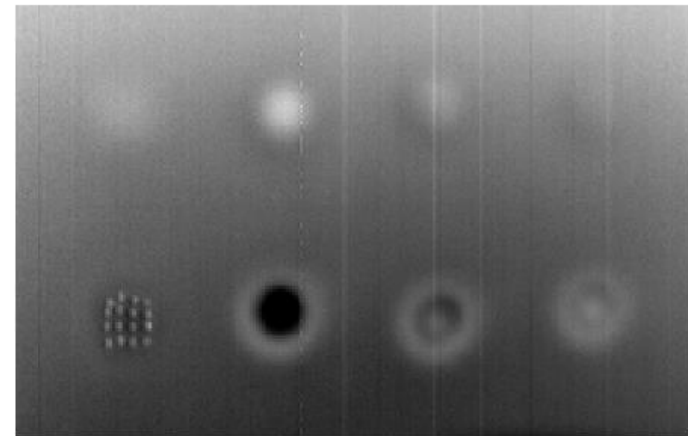

a)

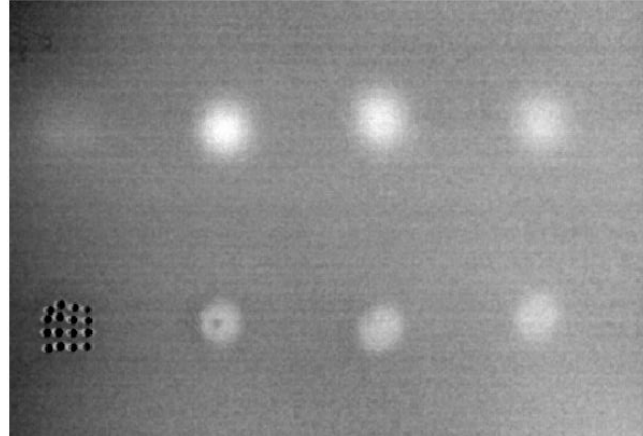

b)

Fig. 7. Phase images obtained in line-scanning TNDT by using optical (a) and inductive (b) heating

\section{Conclusion}

Two-layer structures representing thermal insulation on a metallic base are ubiquitous in the aerospace, petro chemistry and power production. A classical one-sided TNDT of such structures by using surface optical heating may be not optimal for detecting delaminations located at depths greater than $4 \mathrm{~mm}$. In this case, a useful test procedure may involve line-scanning inductive heating of the metallic base that converts the test into two-sided. Such procedure provides a uniform heating pattern and allows efficient estimation of defect lateral size. However, performing defect characterization requires further research in this case.

\section{REFERENCES}

[1] Oswald-Tranta B., Tuschl C., Schledjewski R. "Flash and inductive thermography for CFRP inspection", Proc. SPIE 11004, Thermosense: Thermal Infrared Applications XLI, (2019), doi: 10.1117/12.2520104.

[2] Green D.R. "Emissivity-independent infrared thermal testing method", Mater. Evaluation. - Vol. 23, Issue 2, (1965), pp. 79-85.

[3] Denisov S.S., Volkov Ya.A., Storozhenko V.A. et al. "A scanning optical head for active thermal NDT", Defectoscopiya (in Russian). - Vol. 6, (1975), pp. 116-118.

[4] Chulkov A. O., Nesteruk D. A., Vavilov V. P. et al. "Automated Thermal NDT of Large-Size Parts" NDT World (in Russian). - Vol. 23, no. 1, (2020) pp. 56-59, doi: 10.12737/1609-3178-2020-54-57. 